\title{
4D Near-Field Source Localization Using Cumulant
}

\author{
Junli Liang, ${ }^{1,2}$ Shuyuan Yang, ${ }^{1,2}$ Junying Zhang, ${ }^{3}$ Li Gao, ${ }^{1,2}$ and Feng Zhao ${ }^{4}$ \\ ${ }^{1}$ Institute of Acoustics, Chinese Academy of Sciences, Beijing 100080, China \\ ${ }^{2}$ Graduate School of Chinese Academy of Sciences, Beijing 100039, China \\ ${ }^{3}$ National Laboratory of Radar Signal Processing, Xidian University, Xi'an 710071, China \\ ${ }^{4}$ School of Computer Science and Engineering, Xidian University, Xi'an 710071, China
}

Received 20 September 2006; Revised 1 January 2007; Accepted 24 March 2007

Recommended by Sabine Van Huffel

\begin{abstract}
This paper proposes a new cumulant-based algorithm to jointly estimate four-dimensional (4D) source parameters of multiple near-field narrowband sources. Firstly, this approach proposes a new cross-array, and constructs five high-dimensional Toeplitz matrices using the fourth-order cumulants of some properly chosen sensor outputs; secondly, it forms a parallel factor (PARAFAC) model in the cumulant domain using these matrices, and analyzes the unique low-rank decomposition of this model; thirdly, it jointly estimates the frequency, two-dimensional (2D) directions-of-arrival (DOAs), and range of each near-field source from the matrices via the low-rank three-way array (TWA) decomposition. In comparison with some available methods, the proposed algorithm, which efficiently makes use of the array aperture, can localize $N-3$ sources using $N$ sensors. In addition, it requires neither pairing parameters nor multidimensional search. Simulation results are presented to validate the performance of the proposed method.
\end{abstract}

Copyright (C) 2007 Junli Liang et al. This is an open access article distributed under the Creative Commons Attribution License, which permits unrestricted use, distribution, and reproduction in any medium, provided the original work is properly cited.

\section{INTRODUCTION}

Estimation of directions-of-arrival (DOAs) has received a significant amount of attention over the last several decades. It is a key problem in array signal processing areas such as radar, sonar, radio astronomy, and mobile communication systems. Many classical algorithms have been developed to solve this problem, such as the maximum likelihood (ML) method [1], the MUSIC method [2], and the ESPRIT method [3]. Most of these methods make the assumption that the sources are located relatively far from the array so that the waves emitted by these sources can be considered as plane waves. With such an assumption, each signal wavefront can be characterized by the DOAs of the source [4]. However, when a source is located close to the array (i.e., near field) [5], the wavefront must be characterized by both the DOAs and the range parameters of the source. A good approximation of the nonlinear propagation delay function consists of its second-order Taylor expansion (Fresnel approximation). Using such an approximation, the propagation delay varies quadratically with sensor location, and the range information must be incorporated into the signal model. Therefore, the estimation of the near-field source parameters is more complicated than that of far-field one, and the classical DOAs estimation methods for far-field sources are no longer applicable.

To solve near-field source localization problem, many algorithms were addressed, such as the ML method [5], the 2D MUSIC methods [6-9], the linear prediction methods [10, 11], and the ESPRIT-like methods [12-15]. However, these methods for near-field source localization [5-15] mainly focused on two-dimensional (2D) case, that is, estimating the azimuth and range only. Recently, several algorithms [1618] were addressed to deal with three-dimensional (3D) source localization, which is a joint azimuth, elevation, and range estimation problem. For example, Kabaoglu et al. [16] proposed an expectation-maximization (EM)-based algorithm, in which only a subset of the parameters is estimated iteratively while the other parameters remain fixed. Despite its effectiveness, this algorithm has extremely demanding computational complexity due to the search computation and iteration process. Hung et al. [17] extended the 2D MUSIC method to 3D one, but this method requires a 3D search of the extended cost function. To avoid these search computations, a second-order statistics (SOS)based algorithm was addressed recently in [18], but this 
method, which suffers a heavy loss of the array aperture, can localize not more than $(1 / 4)(N-5)$ sources using $N$ sensors. In addition, it requires a quadratic phase transform algorithm to pair the separately estimated parameters. Note that all these algorithms addressed in [16-18] cannot estimate signal frequencies simultaneously. However, when these frequencies need to be estimated, the 3D nearfield source localization problem actually becomes a fourdimensional (4D) one. Hence it is necessary to develop a joint $4 \mathrm{D}$ parameter estimation algorithm for near-field sources.

The above-mentioned analyses show that the main difficulties of near-field source localization problem consist of: (i) avoiding multidimensional search which results in extremely demanding computational complexity; (ii) reducing the loss of the array aperture; (iii) pairing source parameters (i.e., frequency, azimuth, elevation, and range) so as to localize the near-field sources accurately.

As a useful analysis tool of data arrays, the parallel factor (PARAFAC) model [19-22] is a generalization of low-rank matrix decomposition to three-way arrays (TWAs) or multiway arrays (MWAs). Unlike singular value decomposition, PARAFAC does not impose orthogonality constraints, and relies on certain conditions [23-29] regarding the uniqueness of low-rank TWA (or MWA) decomposition. Because of its direct link to low-rank decomposition, PARAFAC has wide applications in numerous and diverse disciplines [22, $26,30,31]$.

In this paper, we develop a new cumulant-based algorithm for 4D near-field source localization (see [32] for the detailed definition of cumulant). The key point of this paper is to construct five high-dimensional Toeplitz matrices using the cumulants of some properly chosen sensor outputs and form an identifiable PARAFAC model in the fourthorder cumulant domain. The proposed algorithm requires neither pairing parameters nor multidimensional search. In addition, it can efficiently use the array aperture.

The rest of this paper is organized as follows. The signal and PARAFAC models are introduced in Section 2. A 4D near-field source localization algorithm is developed in Section 3. Simulation results are presented in Section 4. Conclusions are drawn in Section 5.

\section{PROBLEM FORMULATION AND PARAFAC MODEL}

\subsection{Problem formulation}

Consider $L$ near-field, narrowband, and independent radiating sources impinging upon a cross array aligned with $\mathbf{x}$ and $\mathbf{y}$ axes, as shown in Figure 1. Each subarray consists of uniformly spaced omnidirectional sensors with inter-element spacing $d$. The $\mathbf{x}$ subarray consists of $2 N$ sensors, while the y subarray is composed of 3 ones. The cross one is chosen as the phase reference point. After being down-converted to baseband and sampled at a proper sampling rate that satisfies the Nyquist rate, the signals received by the $(i, 0)$ th and $(0, m)$ th sensors can be approximately expressed by (see $[14,18]$ for details):

$$
\begin{array}{r}
x_{i, 0}(k)=\sum_{l=1}^{L} s_{l}(k) e^{j \omega_{l} k} e^{j\left(i \gamma_{x l}+i^{2} \phi_{x l}\right)}+n_{i, 0}(k), \\
i=-N+1, \ldots,-1,0,1, \ldots, N, \\
x_{0, m}(k)=\sum_{l=1}^{L} s_{l}(k) e^{j \omega_{l} k} e^{j\left(m \gamma_{y l}+m^{2} \phi_{y l}\right)}+n_{0, m}(k), \\
m=-1,1,
\end{array}
$$

respectively, where $s_{l}(k) e^{j \omega_{l} k}$ denotes the $l$ th source signal with the normalized radian frequency $\omega_{l}$, while $n_{i, 0}(k)$ and $n_{0, m}(k)$ represent the additive measurement noise. In addition, electric angles $\gamma_{x l}, \phi_{x l}, \gamma_{y l}$, and $\phi_{y l}$ are given by

$$
\begin{aligned}
\gamma_{x l} & =-\frac{2 \pi d \sin \alpha_{l} \cos \beta_{l}}{\lambda}, \\
\phi_{x l} & =\frac{\pi d^{2}\left(1-\sin ^{2} \alpha_{l} \cos ^{2} \beta_{l}\right)}{\lambda r_{l}}, \\
\gamma_{y l} & =-\frac{2 \pi d \sin \alpha_{l} \sin \beta_{l}}{\lambda}, \\
\phi_{y l} & =\frac{\pi d^{2}\left(1-\sin ^{2} \alpha_{l} \sin ^{2} \beta_{l}\right)}{\lambda r_{l}},
\end{aligned}
$$

for $l=1, \ldots, L$, respectively, where $\lambda$ is the related propagation wavelength, and $\left\{\alpha_{l}, \beta_{l}, r_{l}\right\}$ denote the azimuth, elevation, and range of the $l$ th source.

The objective of this paper is to jointly estimate the frequency $\omega_{l}$, the 2D DOA $\left\{\alpha_{l}, \beta_{l}\right\}$, and the range $r_{l}$ of the $l$ th source for $l=1, \ldots, L$.

Throughout the rest of the paper, the following hypotheses are assumed to hold.

(H1) The source signals are statistically mutually independent, non-Gaussian, and narrowband stationary processes with nonzero kurtosis.

(H2) The sensor noise is zero-mean Gaussian signal and independent of the source signals.

(H3) The source parameters are different from each other, that is, $\gamma_{x i}+\phi_{x i} \neq \gamma_{x j}+\phi_{x j}, \gamma_{x i}-\phi_{x i} \neq \gamma_{x j}-\phi_{x j}, \gamma_{y i}-\phi_{y i} \neq$ $\gamma_{y j}-\phi_{y j}, \gamma_{y i}+\phi_{y i} \neq \gamma_{y j}+\phi_{y j}$, and $\omega_{i} \neq \omega_{j}$ for $i \neq j$. In fact, this hypothesis can be alleviated, and the detailed analyses are given in Section 3.

(H4) For uniquely identifying $L$ sources, we require $d \leq \lambda / 4$ and $L<2 N$.

\subsection{PARAFAC model $[22,26,30]$}

Definition 1. Consider a $(I \times J \times K)$-dimensional TWA $X=$ $(\mathbf{R} \otimes \mathbf{U}) \mathbf{W}^{T}(\otimes$ stands for Kronecker product) with typical element $x_{i, j, k}$ and the $F$-component trilinear decomposition

$$
x_{i, j, k}=\sum_{f=1}^{F} r_{i, f} u_{j, f} w_{k, f}
$$




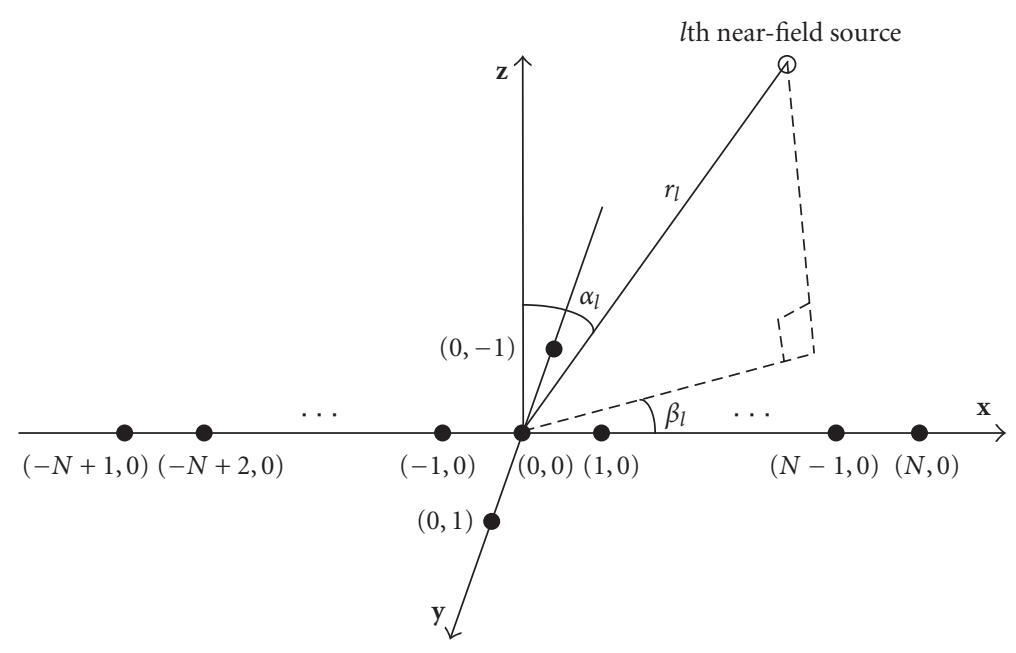

FIGURE 1: proposed cross-array for 4D near-field source localization problem.

for all $i=1, \ldots, I, j=1, \ldots, J$, and $k=1, \ldots, K$, where $r_{i, f}$ represents the $(i, f)$ th element of $(I \times F)$-dimensional matrix R. Similarly, $u_{j, f}$ and $w_{k, f}$ stand for $(j, f)$ th and $(k, f)$ th elements of $(J \times F)$ and $(K \times F)$-dimensional matrices $\mathbf{U}$ and W, respectively. Equation (3) expresses $x_{i, j, k}$ as a sum of $F$ rank-1 triple products; it is known as PARAFAC analysis of $x_{i, j, k}$.

Definition 2. Let $g_{i}(\mathbf{R})$ denote a diagonal matrix composed of the $i$ th row of matrix $\mathbf{R}$, and $g^{-1}(\boldsymbol{\Lambda})$ stands for a row vector made up of the diagonal elements of diagonal matrix $\Lambda$.

In a compact form, $X$ can be expressed in terms of its $2 \mathrm{D}$ slice $X_{i}\left((J \times K)\right.$-dimensional matrix, that is, $\left.X_{i}=\left[x_{i, .,:}\right]\right)$ as

$$
X_{i}=\mathbf{U} g_{i}(\mathbf{R}) \mathbf{W}^{T}, \quad i=1, \ldots, I .
$$

Under certain conditions, $X$ can be decomposed uniquely into matrices $\mathbf{R}, \mathbf{U}$, and $\mathbf{W}$. These conditions are based on the notion of Kruskal-rank [23-26].

Definition 3. The Kruskal rank (or k-rank) [23-26] of matrix $\mathbf{R}$ is $k_{\mathbf{R}}$ if and only if arbitrary $k_{\mathbf{R}}$ columns of $\mathbf{R}$ are linearly independent and either $\mathbf{R}$ has $k_{\mathbf{R}}$ columns or $\mathbf{R}$ contains a set of $k_{\mathbf{R}}+1$ linearly dependent columns. Note that Kruskal rank is always less than or equal to the conventional matrix rank. If $\mathbf{R}$ is of full column rank, then it is also of full $k$-rank.

Theorem 1. Let $X_{i}$ be defined as in (4). R, U, and $\mathbf{W}$ can be recovered uniquely up to permutation and scaling ambiguity, irrespective of whether the elements of $X$ are real values [2325] or complex ones [26], as long as

$$
k_{\mathrm{R}}+k_{\mathrm{U}}+k_{\mathrm{W}} \geq 2 F+2,
$$

which is the well-known Kruskal's condition. In fact, there are different results that guarantee PARAFAC uniqueness under different conditions [27-29]. For instance, Leurgans et al. [27] analyzed the condition for the decomposition of three-way arrays which have rank 1 . While Lathauwer [29] considered the decomposition of higher-order tensors which have the property that the rank is smaller than the greatest dimension.

\section{PROPOSED ALGORITHM}

\subsection{PARAFAC model formulation}

To develop a new joint estimation algorithm, we begin with the $(2 N \times 2 N)$-dimensional cumulant matrix $\mathbf{C}_{1}$, the $(m, n)$ th element of which has the following form:

$$
\mathbf{C}_{1}(m, n)=\sum_{l=1}^{L} c_{4 s l} e^{j\left(\gamma_{x l}+\phi_{x l}\right)} e^{j(m-n)\left(\gamma_{x l}+\phi_{x l}\right)}, \quad 1 \leq m, n \leq 2 N
$$

where $c_{4 s_{l}}=\operatorname{cum}\left(s_{k}(k), s_{l}^{*}(k), s_{l}(k), s_{l}^{*}(k)\right)$ is the fourthorder kurtosis of the $l$ th source. Note that $\mathrm{C}_{1}$ can be represented in a compact form as $\mathbf{C}_{1}=\mathbf{A} \Omega \Lambda \mathbf{C}_{4 s} \mathbf{A}^{H}$, where the superscript $H$ denotes the Hermitian transpose, $\mathbf{C}_{4 s}=$ $\operatorname{diag}\left[c_{4 s_{1}}, c_{4 s_{2}}, \ldots, c_{4 s_{L}}\right], \Omega=\operatorname{diag}\left[e^{j \gamma_{x 1}}, e^{j \gamma_{x 2}}, \ldots, e^{j \gamma_{x L}}\right], \Lambda=$ $\operatorname{diag}\left[e^{j \phi_{x 1}}, e^{j \phi_{x 2}}, \ldots, e^{j \phi_{x L}}\right], \mathbf{A}=\left[\begin{array}{llll}\mathbf{a}_{1} & \mathbf{a}_{2} & \cdots & \mathbf{a}_{L}\end{array}\right]$, and $\mathbf{a}_{l}=$ $\left[1, e^{j\left(\gamma_{x l}+\phi_{x l}\right)}, \ldots, e^{j(2 N-1)\left(\gamma_{x l}+\phi_{x l}\right)}\right]^{T}, l=1, \ldots, L$.

Due to the complicated signal model of near-field sources, it is difficult to derive such a cumulant matrix from the array outputs directly. However, it is easily seen from (6) that the matrix $\mathbf{C}_{1}$ has the same structure as Toeplitz matrices theoretically. It is well known that Toeplitz matrices are matrices having constant entries along their diagonals. Hence we consider approximating $\mathbf{C}_{1}$ by virtue of a set of estimated cumulants.

For different sensor lags, we define a column vector $\mathbf{h}_{1}$, the $i$ th element of which can be represented as

$$
\begin{aligned}
\mathbf{h}_{1}(i, 1) & =\operatorname{cum}\left(x_{0,0}(k), x_{0,0}^{*}(k), x_{(N+1)-i, 0}(k), x_{-N+i, 0}^{*}(k)\right) \\
& =\sum_{l=1}^{L} c_{4 s_{l}} e^{j(2 N-2 i)\left(\gamma_{x l}+\phi_{x l}\right)} e^{j\left(\gamma_{x l}+\phi_{x l}\right)}, \quad i=1,2, \ldots, 2 N,
\end{aligned}
$$

where the superscript $*$ denotes the complex conjugate. It is obvious that the elements of $\mathbf{h}_{1}$ can merely "fill" the $(m, n)$ th position of an approximated matrix, where $(m-n)$ is an even 
number. To construct the whole approximated matrix, we define another column vector $\mathbf{h}_{2}$

$$
\begin{aligned}
\mathbf{h}_{2}(i, 1) & =\operatorname{cum}\left(x_{1,0}(k), x_{0,0}^{*}(k), x_{(N+1)-i, 0}(k), x_{-N+i, 0}^{*}(k)\right) \\
& =\sum_{l=1}^{L} c_{4 s_{l}} e^{j(2 N-2 i+1)\left(\gamma_{x l}+\phi_{x l}\right)} e^{j\left(\gamma_{x l}+\phi_{x l}\right)}, \quad i=1,2, \ldots, 2 N,
\end{aligned}
$$

which can complement the rest of the approximated matrix.

Furthermore, for different sensor and time lags, we define other eight column vectors:

$$
\begin{aligned}
& \mathbf{h}_{3}(i, 1)=\operatorname{cum}\left(x_{0,0}(k), x_{-1,0}^{*}(k), x_{(N+1)-i, 0}(k), x_{-N+i, 0}^{*}(k)\right) \\
& =\sum_{l=1}^{L} c_{4 s_{l}} e^{j(2 N-2 i)\left(\gamma_{x l}+\phi_{x l}\right)} e^{j 2 \gamma_{x l}}, \quad i=1,2, \ldots, 2 N, \\
& \mathbf{h}_{4}(i, 1)=\operatorname{cum}\left(x_{1,0}(k), x_{-1,0}^{*}(k), x_{(N+1)-i, 0}(k), x_{-N+i, 0}^{*}(k)\right) \\
& =\sum_{l=1}^{L} c_{4 s_{l}} e^{j(2 N-2 i+1)\left(\gamma_{x l}+\phi_{x l}\right)} e^{j 2 \gamma_{x l}}, \quad i=1,2, \ldots, 2 N, \\
& \mathbf{h}_{5}(i, 1)=\operatorname{cum}\left(x_{0,0}(k+1), x_{0,0}^{*}(k), x_{(N+1)-i, 0}(k), x_{-N+i, 0}^{*}(k)\right) \\
& =\sum_{l=1}^{L} c_{4 s_{l}} e^{j(2 N-2 i)\left(\gamma_{x l}+\phi_{x l}\right)} e^{j\left(\gamma_{x l}+\phi_{x l}\right)} e^{j \omega_{l}}, \\
& i=1,2, \ldots, 2 N, \\
& \mathbf{h}_{6}(i, 1)=\operatorname{cum}\left(x_{1,0}(k+1), x_{0,0}^{*}(k), x_{(N+1)-i, 0}(k), x_{-N+i, 0}^{*}(k)\right) \\
& =\sum_{l=1}^{L} c_{4 s_{l}} e^{j(2 N-2 i+1)\left(\gamma_{x l}+\phi_{x l}\right)} e^{j\left(\gamma_{x l}+\phi_{x l}\right)} e^{j \omega_{l}}, \\
& i=1,2, \ldots, 2 N \text {, } \\
& \mathbf{h}_{7}(i, 1)=\operatorname{cum}\left(x_{0,0}(k), x_{0,-1}^{*}(k), x_{(N+1)-i, 0}(k), x_{-N+i, 0}^{*}(k)\right) \\
& =\sum_{l=1}^{L} c_{4 s_{l}} e^{j(2 N-2 i)\left(\gamma_{x l}+\phi_{x l}\right)} e^{j\left(\gamma_{x l}+\phi_{x l}\right)} e^{j\left(\gamma_{y l}-\phi_{y l}\right)}, \\
& i=1,2, \ldots, 2 N, \\
& \mathbf{h}_{8}(i, 1)=\operatorname{cum}\left(x_{1,0}(k), x_{0,-1}^{*}(k), x_{(N+1)-i, 0}(k), x_{-N+i, 0}^{*}(k)\right) \\
& =\sum_{l=1}^{L} c_{4 s_{l}} e^{j(2 N-2 i+1)\left(\gamma_{x l}+\phi_{x l}\right)} e^{j\left(\gamma_{x l}+\phi_{x l}\right)} e^{j\left(\gamma_{y l}-\phi_{y l}\right)}, \\
& i=1,2, \ldots, 2 N \\
& \mathbf{h}_{9}(i, 1)=\operatorname{cum}\left(x_{0,0}(k), x_{0,1}^{*}(k), x_{(N+1)-i, 0}(k), x_{-N+i, 0}^{*}(k)\right) \\
& =\sum_{l=1}^{L} c_{4 s_{l}} e^{j(2 N-2 i)\left(\gamma_{x l}+\phi_{x l}\right)} e^{j\left(\gamma_{x l}+\phi_{x l}\right)} e^{j\left(-\gamma_{y l}-\phi_{y l}\right)}, \\
& i=1,2, \ldots, 2 N \\
& \mathbf{h}_{10}(i, 1)=\operatorname{cum}\left(x_{1,0}(k), x_{0,1}^{*}(k), x_{(N+1)-i, 0}(k), x_{-N+i, 0}^{*}(k)\right) \\
& =\sum_{l=1}^{L} c_{4 s_{l}} e^{j(2 N-2 i+1)\left(\gamma_{x l}+\phi_{x l}\right)} e^{j\left(\gamma_{x l}+\phi_{x l}\right)} e^{j\left(-\gamma_{y l}-\phi_{y l}\right)}, \\
& i=1,2, \ldots, 2 N \text {. }
\end{aligned}
$$

Thus, by virtue of these eight column vectors, we can construct four Toeplitz matrices $\mathbf{C}_{2}, \mathbf{C}_{3}, \mathbf{C}_{4}$, and $\mathbf{C}_{5}$ :

$\mathrm{C}_{i}(m, n)$

$$
= \begin{cases}\mathbf{h}_{2 \times i}\left(N-\frac{m-n-1}{2}, 1\right) & \text { if }(m-n) \text { is an odd number, } \\ \mathbf{h}_{2 \times i-1}\left(N-\frac{m-n}{2}, 1\right) & \text { if }(m-n) \text { is an even number } \\ & 1 \leq m, n \leq 2 N, i=2, \ldots, 5 .\end{cases}
$$

It is obvious that these matrices have the following compact forms:

$$
\begin{aligned}
& \mathbf{C}_{2}=\mathbf{A} \Omega^{2} \mathbf{C}_{4 s} \mathbf{A}^{H}, \\
& \mathbf{C}_{3} \cong \mathbf{A} \Omega \Lambda \Phi_{1} \mathbf{C}_{4 s} \mathbf{A}^{H}, \\
& \mathbf{C}_{4}=\mathbf{A} \Omega \Lambda \Phi_{2} \mathbf{C}_{4 s} \mathbf{A}^{H}, \\
& \mathbf{C}_{5}=\mathbf{A} \Omega \Lambda \Phi_{3} \mathbf{C}_{4 s} \mathbf{A}^{H},
\end{aligned}
$$

where

$$
\begin{aligned}
& \boldsymbol{\Phi}_{1}=\operatorname{diag}\left[e^{j \omega_{1}}, e^{j \omega_{2}}, \ldots, e^{j \omega_{L}}\right], \\
& \boldsymbol{\Phi}_{2}=\operatorname{diag}\left[e^{j\left(\gamma_{y 1}-\phi_{y 1}\right)}, e^{j\left(\gamma_{y 2}-\phi_{y 2}\right)}, \ldots, e^{j\left(\gamma_{y L}-\phi_{y L}\right)}\right], \\
& \boldsymbol{\Phi}_{3}=\operatorname{diag}\left[e^{j\left(-\gamma_{y 1}-\phi_{y 1}\right)}, e^{j\left(-\gamma_{y 2}-\phi_{y 2}\right)}, \ldots, e^{j\left(-\gamma_{y L}-\phi_{y L}\right)}\right] .
\end{aligned}
$$

Since all the source signals are assumed to have nonzero kurtosis, $\mathbf{C}_{4 s}$ is an invertible diagonal matrix. Besides, because of the assumptions $\gamma_{x i}+\phi_{x i} \neq \gamma_{x j}+\phi_{x j}$ and $L \leq 2 N$ (see Section 2.1), $\mathbf{A}$ is a Vandermonde matrix with full column rank $L$. Hence, $\mathbf{C}_{1}, \mathbf{C}_{2}, \mathbf{C}_{3}, \mathbf{C}_{4}$, and $\mathbf{C}_{5}$ are all $(2 N \times 2 N)$ dimensional matrices with $\operatorname{rank} L$.

In fact, since the snapshot size is finite, the estimates $\widehat{\mathrm{C}}_{1}$, $\widehat{\mathbf{C}}_{2}, \widehat{\mathbf{C}}_{3}, \widehat{\mathbf{C}}_{4}$, and $\widehat{\mathbf{C}}_{5}$ contain some estimation errors, which can form other five matrices, that is, $V_{1}, V_{2}, V_{3}, V_{4}$, and $V_{5}$. Similar to $(4)$, we define a $(2 N \times 2 N \times 5)$-dimensional TWA $\hat{X}$, the five $2 \mathrm{D}$ slices $((2 N \times 2 N)$-dimensional matrix $)$ of which can be represented as

$$
\begin{aligned}
& \hat{X}_{1}=\hat{\mathbf{C}}_{1}=\mathbf{A} \Omega \Lambda \mathbf{C}_{4 s} \mathbf{A}^{H}+V_{1}, \\
& \hat{X}_{2}=\hat{\mathbf{C}}_{2}=\mathbf{A} \Omega^{2} \mathbf{C}_{4 s} \mathbf{A}^{H}+V_{2}, \\
& \hat{X}_{3}=\hat{\mathbf{C}}_{3}=\mathbf{A} \Omega \Lambda \Phi_{1} \mathbf{C}_{4 s} \mathbf{A}^{H}+V_{3}, \\
& \hat{X}_{4}=\hat{\mathbf{C}}_{4}=\mathbf{A} \Omega \Lambda \Phi_{2} \mathbf{C}_{4 s} \mathbf{A}^{H}+V_{4}, \\
& \hat{X}_{5}=\hat{\mathbf{C}}_{5}=\mathbf{A} \Omega \Lambda \Phi_{3} \mathbf{C}_{4 s} \mathbf{A}^{H}+V_{5} .
\end{aligned}
$$

Note that $\hat{X}$ can be represented in a compact form as

$$
\hat{X}=(\mathbf{R} \otimes \mathbf{U}) \mathbf{W}^{T}+V=X+V
$$

where both $X$ and $V$ are $(2 N \times 2 N \times 5)$-dimensional TWAs, $X=(\mathbf{R} \otimes \mathbf{U}) \mathbf{W}^{T}$, and $V$ consists of $V_{1}, V_{2}, V_{3}, V_{4}$, and $V_{5}$. 
In addition, $\mathbf{W}=\mathbf{A}^{*}, \mathbf{U}=\mathbf{A}$, and

$$
\mathbf{R}=\left[\begin{array}{c}
g^{-1}\left(\Omega \Lambda \mathbf{C}_{4 s}\right) \\
g^{-1}\left(\Omega^{2} \mathbf{C}_{4 s}\right) \\
g^{-1}\left(\Omega \Lambda \Phi_{1} \mathbf{C}_{4 s}\right) \\
g^{-1}\left(\Omega \Lambda \Phi_{2} \mathbf{C}_{4 s}\right) \\
g^{-1}\left(\Omega \Lambda \Phi_{3} \mathbf{C}_{4 s}\right)
\end{array}\right] .
$$

It can be seen that the hypothesis (H3) in Section 2.1 can enable $X$ to certainly meet Theorem 1 . In fact, this demanding hypothesis can be alleviated so that this theorem still holds under the following general assumption. Assume these two hypotheses to hold: (i) to any two sources, $\gamma_{x i}+\phi_{x i} \neq$ $\gamma_{x j}+\phi_{x j}$ for $i \neq j$; (ii) not less than two sources have either different $\omega_{i}$, or different $\gamma_{x i}-\phi_{x i}$, or different $\gamma_{y i}-\phi_{y i}$, or different $\gamma_{y i}+\phi_{y i}$. Note that the first hypothesis can guarantee that $k_{\mathrm{W}}=L$ and $k_{\mathrm{U}}=L$, while the second one ensures $k_{\mathrm{R}} \geq 2$, and thus $X$ still satisfies Theorem 1 under this general assumption. In fact, this result holds for one source case, that is, $L=1$, irrespective of these two hypotheses, as long as $X$ does not contain an identically zero 2D slice along any dimension $[22,26]$. In the actual implementation, $X$ is approximated by $\hat{X}$.

\subsection{Description of the proposed algorithm}

As one of the methods for fitting PARAFAC model, trilinear alternating least square (TALS) approach [26, 30, 31, 3336] (other methods [37-39] also can be used to deal with this fitting problem, such as the TALAE method proposed in [37]) is appealing primarily because it is guaranteed to converge monotonically but also because of its relative simplicity (no parameter to tune, and each step solves a standard least square problem) and good performance $[22,35]$. In addition, this method also allows easy incorporation of weighted loss function, missing values, and constraints on some or all of the factors $[22,36]$. The basic idea behind this method for PARAFAC model fitting is to update a subset of parameters using least squares regression every time while keeping the other previous parameter estimates fixed. Such an alternating projections-type procedure is iterated for all subsets of parameters until the convergence is achieved. The computational complexity per iteration $[26,31]$ is equal to the cost of computing a matrix pseudoinverse, that is, $O\left(F^{3}+I J K F\right)$, where $I, J, K$, and $F$ are defined in Section 2.2. Note that when $F$ is small relative to $I, J$, and $K$, only a few iterations are usually required to achieve convergence.

In this paper, we use the COMFAC algorithm $[26,33,34]$ to fit the PARAFAC model. This algorithm is essentially a fast implementation of TALS, and speeds up the least squares fitting procedure by working with a compressed version of the data, thereby avoiding brute-force implementation of alternating least square in the raw data space. It consists of three main parts: (i) compression; (ii) initialization and fitting of PARAFAC in compressed space; (iii) decompression and refinement in the raw data space. The COMFAC MATLAB function described in [34] has such a form $[\mathbf{R}, \mathbf{U}, \mathbf{W}, \bullet, i]=$ $\operatorname{comfac}(\hat{X}, f, \bullet, \bullet, \bullet, \bullet)$, where inputs $\hat{X}$ and $f$, respectively, stand for the decomposing TWA and the corresponding factor number (in this paper, it represents the source number), while outputs $\{\mathbf{R}, \mathbf{U}, \mathbf{W}\}$ and $i$ represent the identification results (matrices) and the iteration number required for the low-rank decomposition. In addition, $\bullet$ denote some other options (see [34] for details). Thus the proposed method can be described as follows.

Step 1. Estimate the cumulant matrices $\widehat{\mathbf{C}}_{1}, \widehat{\mathbf{C}}_{2}, \widehat{\mathbf{C}}_{3}, \widehat{\mathbf{C}}_{4}$, and $\widehat{\mathrm{C}}_{5}$, then construct TWA $\hat{X}$.

Step 2. Implement the COMFAC MATLAB function $[\mathbf{R}, \mathbf{U}$, $\mathbf{W}, \bullet, i]=\operatorname{comfac}(\hat{X}, f, \bullet, \bullet, \bullet, \bullet)$ to fit the PARAFAC model $\hat{X}$, and get the estimates $\widehat{\mathbf{R}}, \hat{\mathbf{U}}$, and $\widehat{\mathbf{W}}$.

Step 3. The estimates of $e^{j\left(\gamma_{x l}+\phi_{x l}\right)}, e^{j\left(\gamma_{x l}-\phi_{x l}\right)}, e^{j\left(-\gamma_{y l}-\phi_{y l}\right)}$, $e^{j\left(\gamma_{y l}-\phi_{y l}\right)}$, and $\omega_{l}$ can be obtained from $\widehat{\mathbf{R}}, \widehat{\mathbf{U}}$, and $\widehat{\mathbf{W}}$ :

$$
\begin{aligned}
\eta_{1, l}= & e^{j\left(\hat{\gamma}_{x l}+\hat{\phi}_{x l}\right)} \\
= & \frac{1}{2(2 N-1)}\left\{\sum_{i=1}^{2 N-1} \frac{\hat{\mathbf{U}}(i+1, l)}{\widehat{\mathbf{U}}(i, l)}+\sum_{i=1}^{2 N-1} \frac{\widehat{\mathbf{W}}^{*}(i+1, l)}{\widehat{\mathbf{W}}^{*}(i, l)}\right\} \\
\eta_{2, l}=e^{j\left(\hat{\gamma}_{x l}-\hat{\phi}_{x l}\right)}= & \frac{\hat{\mathbf{R}}(2, l)}{\widehat{\mathbf{R}}(1, l)}, \\
\eta_{3, l}=e^{j\left(\hat{\gamma}_{y l}-\hat{\phi}_{y l}\right)}= & \frac{\hat{\mathbf{R}}(4, l)}{\widehat{\mathbf{R}}(1, l)}, \\
\eta_{4, l}=e^{j\left(-\hat{\gamma}_{y l}-\hat{\phi}_{y l}\right)}= & \frac{\hat{\mathbf{R}}(5, l)}{\widehat{\mathbf{R}}(1, l)}, \\
& \hat{\omega}_{l}=\angle\left(\frac{\hat{\mathbf{R}}(3, l)}{\widehat{\mathbf{R}}(1, l)}\right)
\end{aligned}
$$

for $l=1, \ldots, L$, respectively.

Step 4. From (16), we can obtain the estimates of $\left\{\gamma_{x l}, \gamma_{y l}\right.$, $\left.\phi_{x l}\right\}$ :

$$
\begin{aligned}
& \hat{\gamma}_{x l}=\frac{\angle\left(\eta_{1, l} \eta_{2, l}\right)}{2}, \\
& \hat{\phi}_{x l}=\frac{\angle\left(\eta_{1, l} / \eta_{2, l}\right)}{2}, \\
& \hat{\gamma}_{y l}=\frac{\angle\left(\eta_{3, l} / \eta_{4, l}\right)}{2} .
\end{aligned}
$$

Step 5. Thus, we can obtain the estimates of $\left\{\alpha_{l}, \beta_{l}\right\}$ and $r_{l}$ :

$$
\begin{aligned}
& \hat{\alpha}_{l}=\operatorname{asin}\left(\frac{\lambda}{2 \pi d} \sqrt{\hat{\gamma}_{x l}^{2}+\hat{\gamma}_{y l}^{2}}\right), \\
& \hat{\beta}_{l}=\operatorname{atan}\left(\frac{\hat{\gamma}_{y l}}{\hat{\gamma}_{x l}}\right), \\
& \hat{r}_{l}=\frac{\pi d^{2}}{\lambda \hat{\phi}_{x l}}\left(1-\sin ^{2} \hat{\alpha}_{l} \cos ^{2} \hat{\beta}_{l}\right),
\end{aligned}
$$

for $l=1, \ldots, L$, respectively. 
Since matrix estimates $\widehat{\mathbf{R}}, \widehat{\mathbf{U}}$, and $\widehat{\mathbf{W}}$ are simultaneously obtained from the low-rank decomposition of $\hat{X}$, and their respective elements, which come from the columns with the same sequence number, are the functions of the parameters of the same source, the proposed algorithm avoids extra pairing computation. However, the method addressed in [18] needs to decompose each matrix respectively, and thus requires a complicated quadratic phase transform method to pair the separately estimated parameters.

Since it can construct five $(2 \mathrm{~N} \times 2 \mathrm{~N})$-dimensional matrices using $2 N+2$ sensors, our algorithm can localize $2 N-1$ sources. However, the method developed in [18] can construct six $([(1 / 2)(N+1)] \times[(1 / 2)(N+1)])$-dimensional matrices using $2 N+3$ sensors (since the algorithm in [18] has a symmetric cross array configuration, we arrange such a cross array of $2 N+3$ sensors for this algorithm), and can localize not more than $(1 / 2)(N-1)$ sources. Regarding the main computational complexity, we only consider the multiplications involved in calculating the matrices and in performing the low-rank TWA decomposition (or the matrix eigendecomposition in [18]). The method in [18] requires calculating four $(N+1)$-dimensional vectors to construct six $([(1 / 2)(N+1)] \times[(1 / 2)(N+1)])$-dimensional SOS matrices, so it requires $O\{4(N+1) m\}$. However, our algorithm requires calculating ten $2 \mathrm{~N}$-dimensional cumulant vectors to construct five $(2 N \times 2 N)$-dimensional Toeplitz matrices, so it requires $O\{180 \mathrm{Nm}$. Relative to the computational complexity from the matrix decomposition (or the lowrank TWA decomposition in our algorithm), the method in [18] decomposes two $([(3 / 2)(N+1)] \times[(1 / 2)(N+1)])$ dimensional matrices separately, so it requires $O\{(9 / 8)(N+$ $\left.1)^{3}\right\}$ and our algorithm uses the COMFAC algorithm to fit a $(2 N \times 2 N \times 5)$-dimensional TWA, and thus the computational complexity per iteration is $O\left\{L^{3}+20 N^{2} L\right\}$. For the simulations in Section 4, only 2 iterations are required to achieve convergence. Hence the total computational complexity of our algorithm is $O\left\{180 \mathrm{Nm}+2\left(L^{3}+20 N^{2} L\right)\right\}$, and is larger than that of [18] (i.e., $\left.O\left\{4(N+1) m+(9 / 8)(N+1)^{3}\right\}\right)$ in the case of $m \gg N$, where $m, 2 N+2$, and $L$ stand for the snapshot, sensor, and source number, respectively.

\section{SIMULATION RESULTS}

Some simulations are conducted in this section to assess the proposed algorithm. We consider a 12-element cross array with element spacing $d=(\lambda / 4)$, as shown in Figure 1. Two equal-power, statistically independent narrow-band sources (bandwidth $=25 \mathrm{kHz}$ ), respectively with center frequency 2.0 and $2.5 \mathrm{MHz}$, radiate on the cross array. The sampling rate is $20 \mathrm{MHz}$ and the received signals are polluted by zero-mean additive white Gaussian noises. The two sources are located at $\left\{\alpha_{1}=5^{\circ}, \beta_{1}=30^{\circ}, r_{1}=1.5 \lambda\right\}$ and $\left\{\alpha_{2}=50^{\circ}, \beta_{2}=\right.$ $\left.15^{\circ}, r_{2}=0.3 \lambda\right\}$, respectively. For comparison, we simultaneously execute the algorithm in [18] which assumes the frequencies are known. Since the algorithm in [18] uses a symmetric cross array, we arrange such an array of 13 sensors for this algorithm. The DOAs, frequency, and range estimates are scaled in units of $\mathrm{rad}, \mathrm{rad} / \mathrm{s}$, and wavelength, respectively,

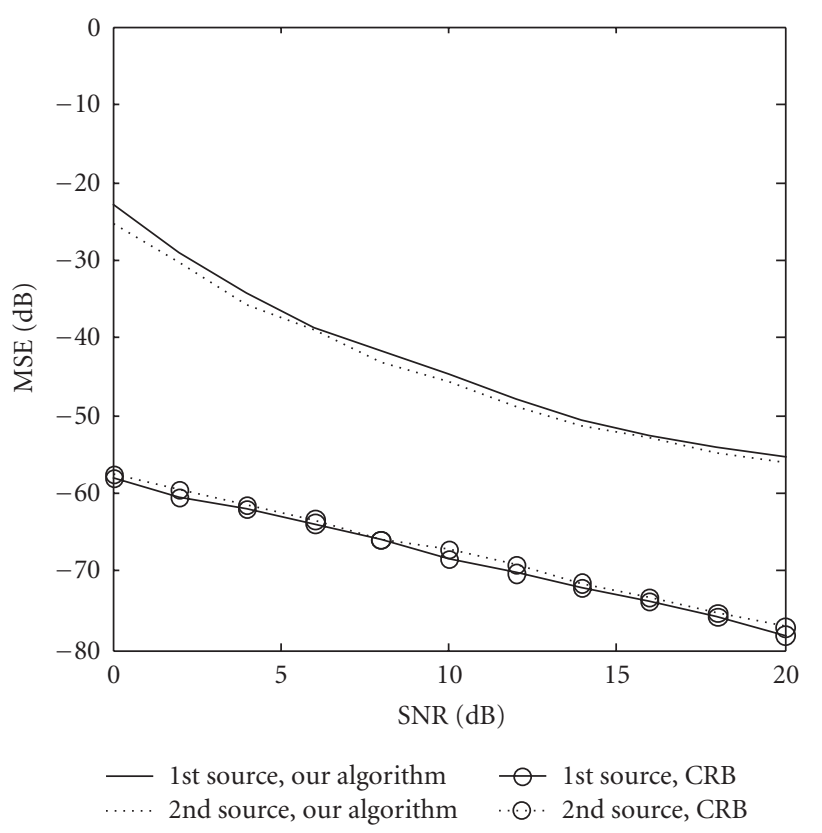

FIGURE 2: Estimation MSE of the frequencies versus input SNR.

and the performance of these algorithms is measured by the mean-square error (MSE) of the estimated parameters. 200 independent Monte Carlo runs are performed to evaluate the estimation errors. At the same time the Cramer-Rao bounds (CRB) for estimating source parameters are obtained from the inverse of Fisher information matrix [1], and shown in the relevant figures.

For the following experiments, we use the short version $[\mathbf{R}, \mathbf{U}, \mathbf{W}, \bullet, i]=\operatorname{comfac}(\hat{X}, 2)$ of COMFAC algorithm $[33,34]$ to fit the $(10 \times 10 \times 5)$-dimensional TWA. In the COMFAC algorithm, we implement the initialization using DTLD function, and employ data compression using the Tucker3 three-way model [40, 41]. For these simulations, only 2 iterations are required to achieve convergence.

In the first experiment, the effect of signal-to-noise (SNR) on the performance of the proposed algorithm is investigated. The snapshot number is set equal to 400 , and the SNR varies from $0 \mathrm{~dB}$ to $20 \mathrm{~dB}$. Figures $2,3,4$, and 5 show the MSE of the frequency, azimuth, elevation, and range estimates of the two sources, respectively.

In the second experiment, the influence of snapshot number on the performance of the proposed algorithm is investigated. The SNR is set equal to $10 \mathrm{~dB}$, and the snapshot number varies from 200 to 2000. Figures 6, 7, 8, and 9 show the MSE of the frequency, azimuth, elevation, and range estimates of the two sources, respectively.

From these simulations, we can arrive at the following conclusion.

(i) Our algorithm has a satisfactory frequency estimation accuracy even at low SNR region, while that of [18] is based on the assumption that the frequencies are known. 


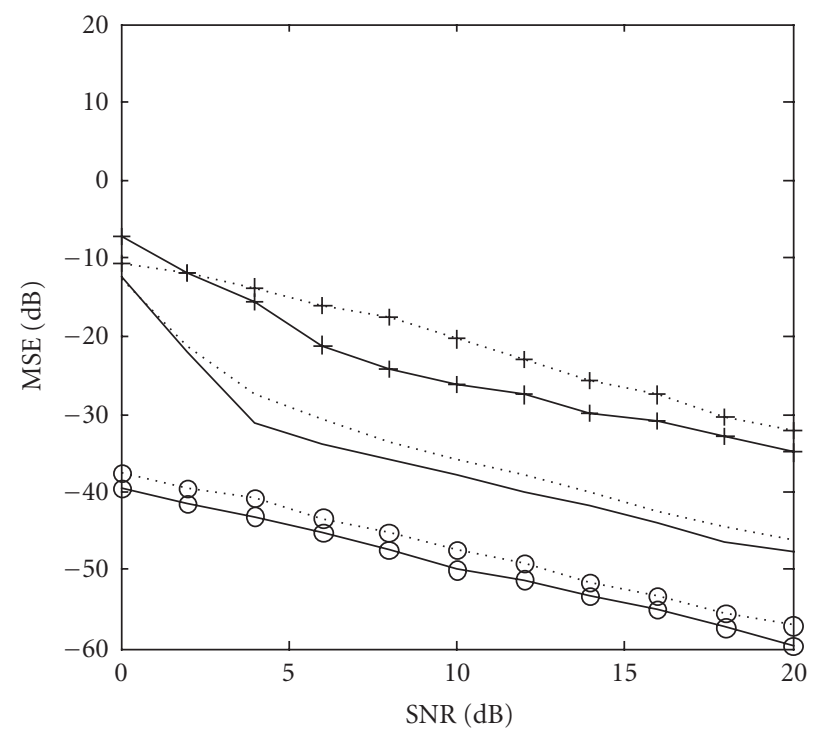

$\begin{array}{llll} & \text { 1st source, our algorithm } & \cdots+\cdot & \text { 2nd source, [18] } \\ \ldots \ldots & \text { 2nd source, our algorithm } & - & \text { 1st source, CRB } \\ + & \text { 1st source, [18] } & \bigcirc & \text { 2nd source, CRB }\end{array}$

FIgURE 3: Estimation MSE of the azimuths versus input SNR.

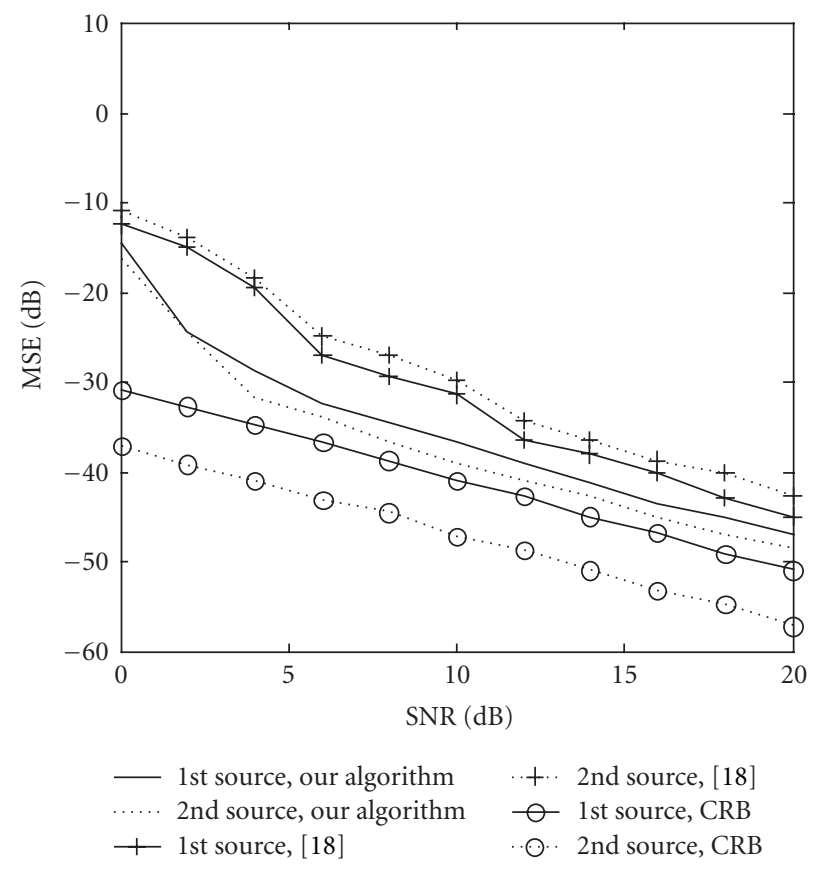

FIGURE 4: Estimation MSE of the elevations versus input SNR.

(ii) Our algorithm has higher estimation accuracy than that of [18].

(iii) The MSE of the range estimate of the 2nd source (closer to the array) is much lower than that of the 1st source.

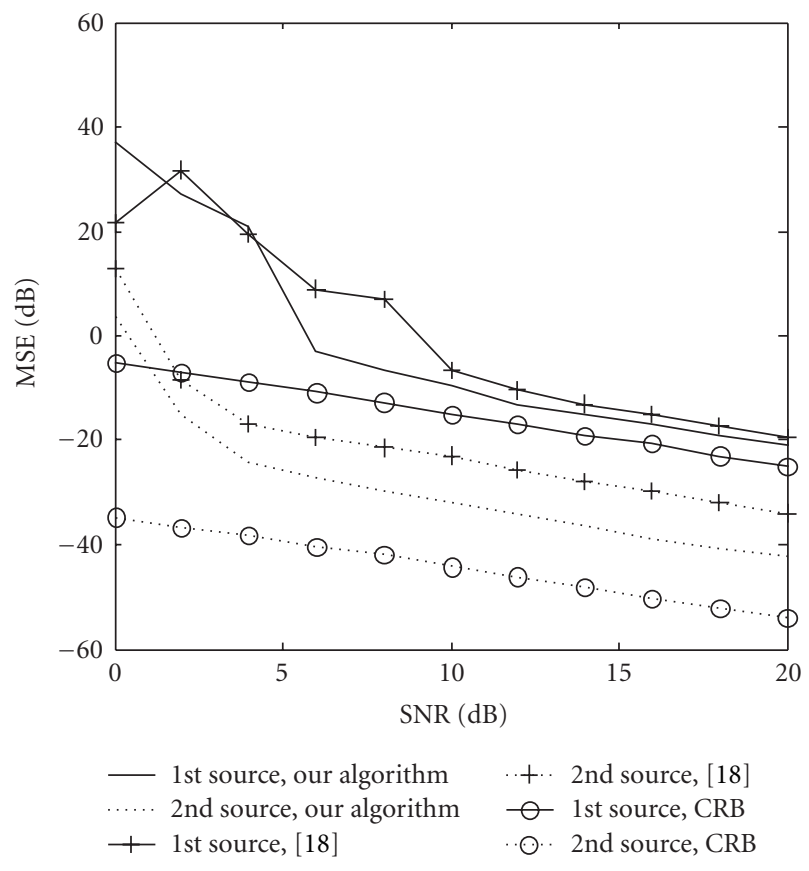

FIgURE 5: Estimation MSE of the ranges versus input SNR.

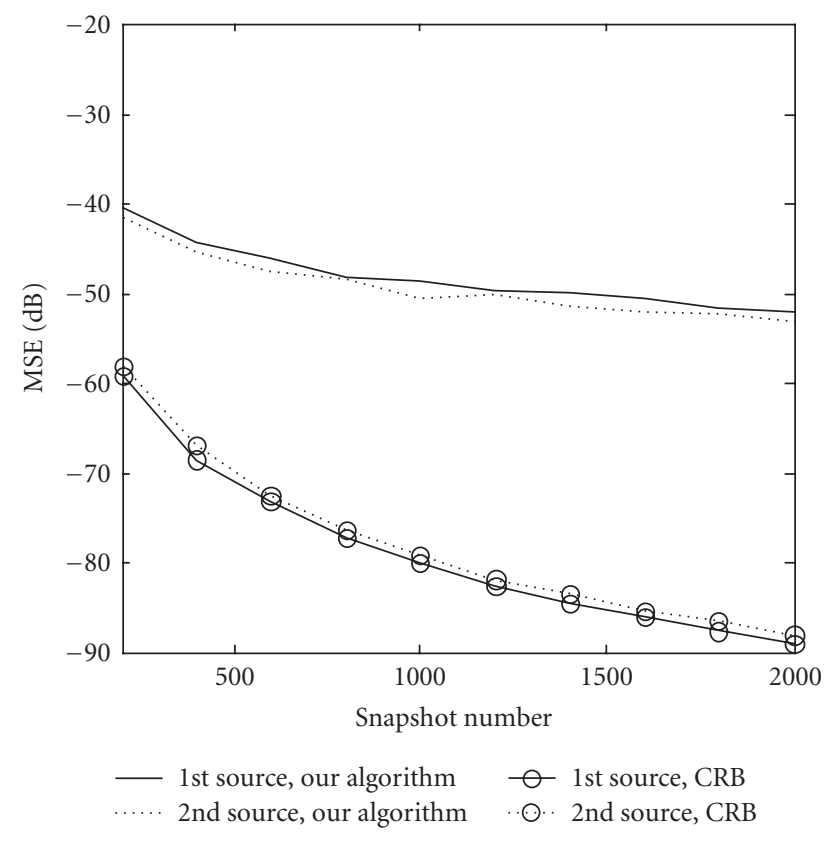

FIGURE 6: Estimation MSE of the frequencies versus snapshot number.

\section{CONCLUSION}

A new approach is proposed for the joint frequencyazimuth-elevation-range estimation of multiple near-field narrowband sources. Based on the characteristics of Toeplitz 


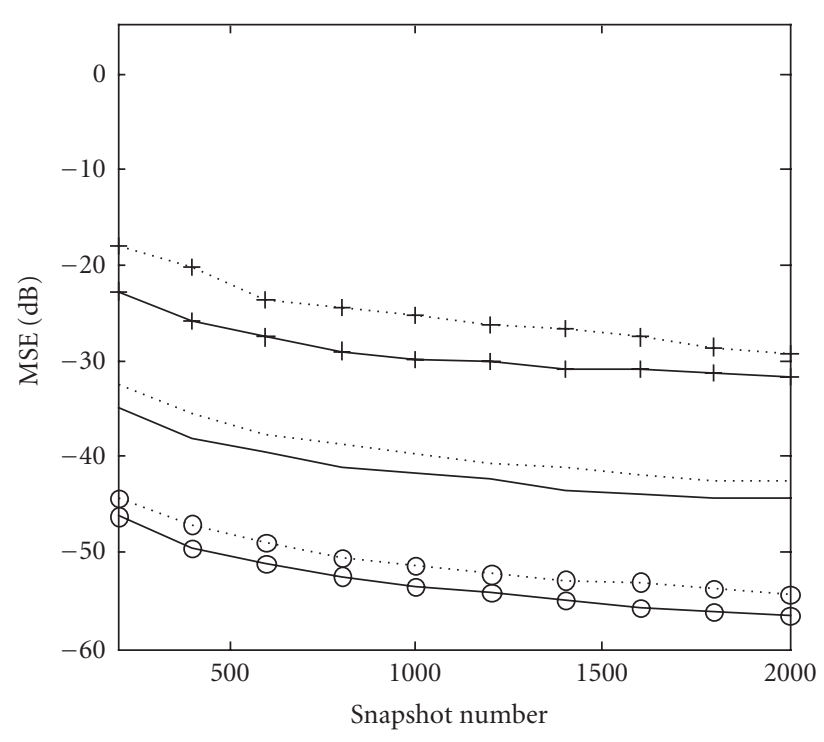

\begin{tabular}{llll}
\hline & 1st source, our algorithm & $\ldots+\cdot$ & 2nd source, [18] \\
$\ldots \ldots$ & 2nd source, our algorithm & - & 1st source, CRB \\
+ & 1st source, $[18]$ & $\bigcirc$ & 2nd source, CRB
\end{tabular}

FIGURE 7: Estimation MSE of the azimuths versus snapshot number.

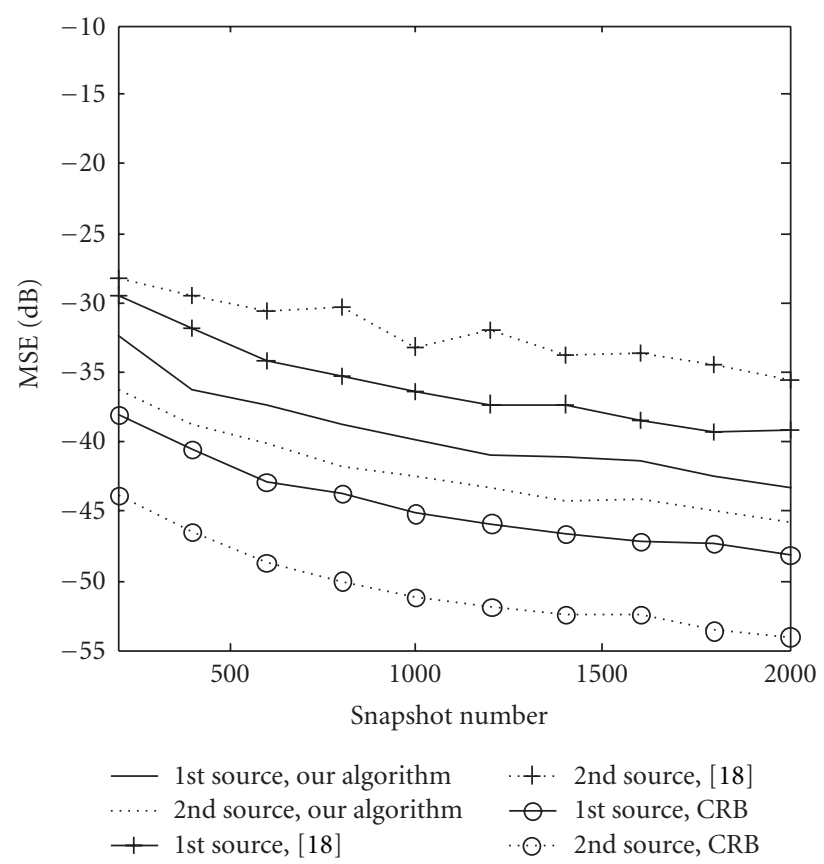

FIGURE 8: Estimation MSE of the elevations versus snapshot number.

matrices, this paper constructs five high-dimensional Toeplitz matrices using some properly chosen cumulants of array outputs so that these matrices can form an identifiable PARAFAC model. The source parameters can be estimated from the matrices via the low-rank decomposition of the model. In comparison with some available methods, the

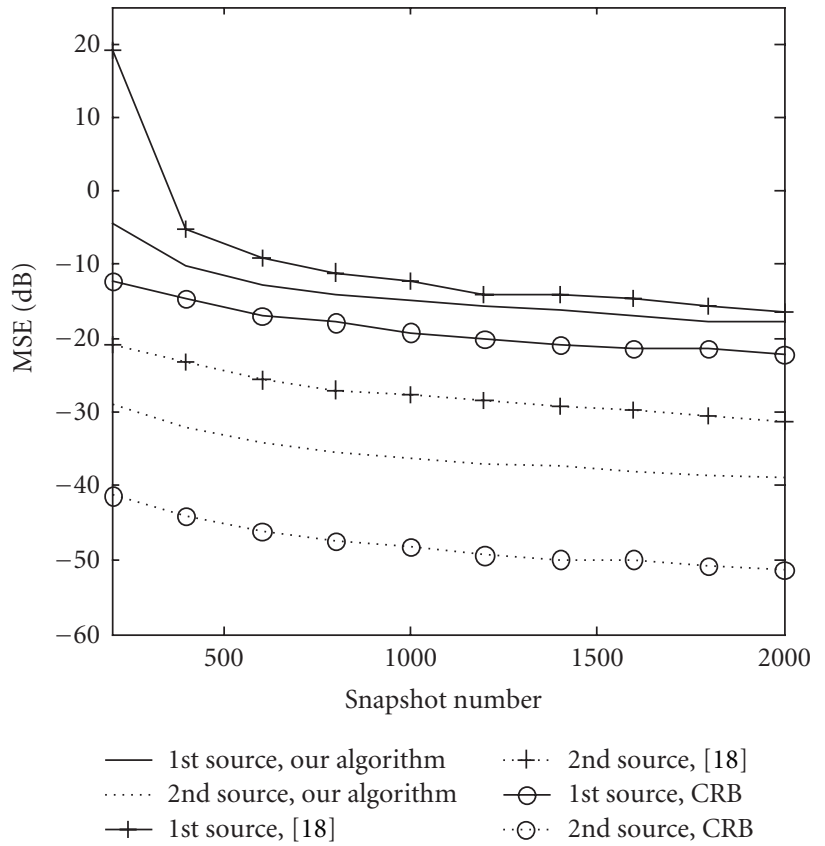

FIgURE 9: Estimation MSE of the ranges versus snapshot number.

proposed approach requires neither pairing parameters nor searching spectral peaks, and can effectively use the array aperture, and thus have higher estimation accuracy under the equivalent sensor number.

\section{ACKNOWLEDGMENTS}

The authors would like to thank the anonymous reviewers, editors Ali H. Sayed and S. Van Huffel for their valuable comments and suggestions on their manuscript.

\section{REFERENCES}

[1] S. M. Kay, Fundamentals of Statistical Signal Processing: Estimation Theory, Prentice-Hall, Upper Saddle River, NJ, USA, 1993.

[2] R. O. Schmidt, "Multiple emitter location and signal parameter estimation," IEEE Transactions on Antennas and Propagation, vol. 34, no. 3, pp. 276-280, 1986.

[3] R. Roy and T. Kailath, "ESPRIT_-estimation of signal parameters via rotational invariance techniques," IEEE Transactions on Acoustics, Speech, and Signal Processing, vol. 37, no. 7, pp. 984-995, 1989.

[4] H. Krim and M. Viberg, "Two decades of array signal processing research: the parametric approach," IEEE Signal Processing Magazine, vol. 13, no. 4, pp. 67-94, 1996.

[5] A. L. Swindlehurst and T. Kailath, "Passive direction-of-arrival and range estimation for near-field sources," in Proceedings of the 4th Annual ASSP Workshop on Spectrum Estimation and Modeling, pp. 123-128, Minneapolis, Minn, USA, August 1988.

[6] Y.-D. Huang and M. Barkat, "Near-field multiple source localization by passive sensor array," IEEE Transactions on Antennas and Propagation, vol. 39, no. 7, pp. 968-975, 1991. 
[7] R. Jeffers, K. L. Bell, and H. L. Van Trees, "Broadband passive range estimation using MUSIC," in Proceedings of IEEE International Conference on Acoustics, Speech, and Signal Processing (ICASSP '02), vol. 3, pp. 2921-2924, Orlando, Fla, USA, May 2002.

[8] A. J. Weiss and B. Friedlander, "Range and bearing estimation using polynomial rooting," IEEE Journal of Oceanic Engineering, vol. 18, no. 2, pp. 130-137, 1993.

[9] D. Starer and A. Nehorai, "Passive localization on near-field sources by path following," IEEE Transactions on Signal Processing, vol. 42, no. 3, pp. 677-680, 1994.

[10] E. Grosicki, K. Abed-Meraim, and Y. Hua, "A weighted linear prediction method for near-field source localization," IEEE Transactions on Signal Processing, vol. 53, no. 10, part 1, pp. 3651-3660, 2005.

[11] K. Abed-Meraim, Y. Hua, and A. Belouchrani, "Second-order near-field source localization: algorithm and performance analysis," in Proceedings of the 30th Asilomar Conference on Signals, Systems, and Computers, vol. 1, pp. 723-727, Pacific Grove, Calif, USA, November 1996.

[12] R. N. Challa and S. Shamsunder, "High-order subspace based algorithms for passive localization of near-field sources," in Proceedings of the 29th Asilomar Conference on Signals, Systems, and Computers, vol. 2, pp. 777-781, Pacific Grove, Calif, USA, October 1995.

[13] N. Yuen and B. Friedlander, "Performance analysis of higher order ESPRIT for localization of near-field sources," IEEE Transactions on Signal Processing, vol. 46, no. 3, pp. 709-719, 1998.

[14] J.-F. Chen, X.-L. Zhu, and X.-D. Zhang, "A new algorithm for joint range-DOA-frequency estimation of near-field sources," EURASIP Journal on Applied Signal Processing, vol. 2004, no. 3, pp. 386-392, 2004.

[15] Y. Wu, L. Ma, C. Hou, G. Zhang, and J. Li, "Subspace-based method for joint range and DOA estimation of multiple nearfield sources," Signal Processing, vol. 86, no. 8, pp. 2129-2133, 2006.

[16] N. Kabaoglu, H. A. Cirpan, E. Cekli, and S. Paker, "Maximum likelihood 3-D near-field source localization using the EM algorithm," in Proceedings of the 8th IEEE International Symposium on Computers and Communication (ISCC '03), vol. 1, pp. 492-497, Kiris-Kemer, Turkey, June-July 2003.

[17] H.-S. Hung, S.-H. Chang, and C.-H. Wu, "3-D MUSIC with polynomial rooting for near-field source localization," in Proceedings of IEEE International Conference on Acoustics, Speech, and Signal Processing (ICASSP '96), vol. 6, pp. 3065-3068, Atlanta, Ga, USA, May 1996.

[18] K. Abed-Meraim and Y. Hua, "3-D near field source localization using second order statistics," in Proceedings of the 31st Asilomar Conference on Signals, Systems, and Computers, vol. 2, pp. 1307-1311, Pacific Grove, Calif, USA, November 1997.

[19] R. B. Cattell, “"Parallel proportional profiles” and other principles for determining the choice of factors by rotation," Psychometrika, vol. 9, no. 4, pp. 267-283, 1944.

[20] J. D. Carroll and J. Chang, "Analysis of individual differences in multidimensional scaling via an n-way generalization of "Eckart-Young" decomposition," Psychometrika, vol. 35, no. 3, pp. 283-319, 1970.

[21] R. A. Harshman, "Foundations of the PARAFAC procedure: models and conditions for an "explanatory" multi-modal factor analysis," UCLA Working Papers in Phonetics, vol. 16, pp. $1-84,1970$.
[22] A. Smilde, R. Bro, and P. Geladi, Multi-Way Analysis with Applications in the Chemical Sciences, John Wiley \& Sons, Chichester, UK, 2004.

[23] J. B. Kruskal, “Three-way arrays: rank and uniqueness of trilinear decompositions, with application to arithmetic complexity and statistics," Linear Algebra and Its Applications, vol. 18, no. 2, pp. 95-138, 1977.

[24] J. B. Kruskal, "Rank decomposition, and uniqueness for 3-way and n-way arrays," in Multiway Data Analysis, R. Coppi and S. Bolasco, Eds., pp. 7-18, North-Holland, Amsterdam, The Netherlands, 1988.

[25] T. Jiang and N. D. Sidiropoulos, "Kruskal's permutation lemma and the identification of CANDECOMP/PARAFAC and bilinear models with constant modulus constraints," IEEE Transactions on Signal Processing, vol. 52, no. 9, pp. 2625-2636, 2004.

[26] N. D. Sidiropoulos, G. B. Giannakis, and R. Bro, "Blind PARAFAC receivers for DS-CDMA systems," IEEE Transactions on Signal Processing, vol. 48, no. 3, pp. 810-823, 2000.

[27] S. E. Leurgans, R. T. Ross, and R. B. Abel, "A decomposition for three-way arrays," SIAM Journal on Matrix Analysis and Applications, vol. 14, no. 4, pp. 1064-1083, 1993.

[28] E. Sanchez and B. R. Kowalski, "Tensorial resolution: a direct trilinear decomposition," Journal of Chemometrics, vol. 4, no. 1, pp. 29-45, 1990.

[29] L. De Lathauwer, "A link between the canonical decomposition in multilinear algebra and simultaneous matrix diagonalization," SIAM Journal on Matrix Analysis and Applications, vol. 28, no. 3, pp. 642-666, 2006.

[30] N. D. Sidiropoulos, R. Bro, and G. B. Giannakis, "Parallel factor analysis in sensor array processing," IEEE Transactions on Signal Processing, vol. 48, no. 8, pp. 2377-2388, 2000.

[31] Y. Rong, S. A. Vorobyov, A. B. Gershman, and N. D. Sidiropoulos, "Blind spatial signature estimation via time-varying user power loading and parallel factor analysis," IEEE Transactions on Signal Processing, vol. 53, no. 5, pp. 1697-1710, 2005.

[32] J. M. Mendel, “Tutorial on higher-order statistics (spectra) in signal processing and system theory: theoretical results and some applications," Proceedings of the IEEE, vol. 79, no. 3, pp. 278-305, 1991.

[33] R. Bro, N. D. Sidiropoulos, and G. B. Giannakis, "A fast least squares algorithm for separating trilinear mixtures," in Proceedings of the 1st International Workshop on Independent Component Analysis and Blind Signal Separation, pp. 289-294, Aussois, France, January 1999.

[34] N. D. Sidiropoulos, "COMFAC: Matlab code for LS fitting of the complex PARAFAC model in 3-D," 1998, http://www.telecom.tuc.gr/ nikos.

[35] R. Bro, "PARAFAC: tutorial and applications," Chemometrics and Intelligent Laboratory Systems, vol. 38, no. 2, pp. 149-171, 1997.

[36] R. Bro and N. D. Sidiropoulos, "Least squares algorithms under unimodality and non-negativity constraints," Journal of Chemometrics, vol. 12, no. 4, pp. 223-247, 1998.

[37] S. A. Vorobyov, Y. Rong, N. D. Sidiropoulos, and A. B. Gershman, "Robust iterative fitting of multilinear models," IEEE Transactions on Signal Processing, vol. 53, no. 8, pp. 2678-2689, 2005.

[38] L. De Lathauwer, B. De Moor, and J. Vandewalle, "Computation of the canonical decomposition by means of a simultaneous generalized schur decomposition," SIAM Journal on 
Matrix Analysis and Applications, vol. 26, no. 2, pp. 295-327, 2004.

[39] G. Tomasi, Practical and computational aspects in chemometric data analysis, Ph.D. thesis, Department of Food Science, Faculty of Life Sciences, University of Copenhagen, Frederiksberg, Denmark, 2006, http://www.models.kvl.dk/research/ theses/.

[40] L. R. Tucker, "The extension of factor analysis to threedimensional matrices," in Contributions to Mathematical Psychology, H. Gulliksen and N. Frederiksen, Eds., pp. 109-127, Holt, Rinehart \& Winston, New York, NY, USA, 1964.

[41] L. R. Tucker, "Some mathematical notes on three-mode factor analysis," Psychometrika, vol. 31, no. 3, pp. 279-311, 1966.

Junli Liang was born in China in 1978. He received his B.S. and M.S. degrees in computer science and technology in Xidian University, in 2001 and 2004, respectively. Currently, he is working towards his Ph.D. degree in Institute of Acoustics, Chinese Academy of Sciences. His research interests include array signal processing, adaptive filtering, pattern recognition, image processing, and intelligent signal processing.

Shuyuan Yang was born in China in 1942. He received his B.S. degree from the HarBin Engineering University in 1968. Currently, he is with the Institute of Acoustics, Chinese Academy of Sciences, Beijing, China, as a Research Fellow. His research interests include digital signal processing, image processing and pattern recognition, and VLSI signal processing.

Junying Zhang was born in China in 1961. She received her Ph.D. degree in signal and information processing from Xidian University, Xi'an, China, in 1998. From 2001 to 2002, she was a Visiting Scholar at the Department of Electrical Engineering and Computer Science, the Catholic University of America, Washington, DC, USA. She is currently a Professor in the School of Computer Science and Engineering in Xidian
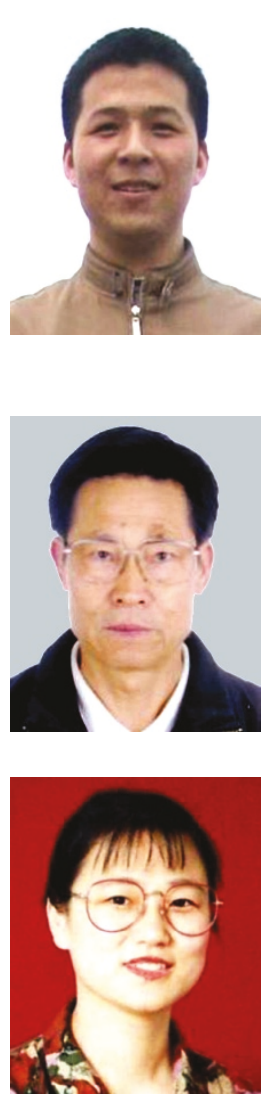

University, Xi'an, China and presently is a Short-Time Research Professor in the Bradley Department of Electrical and Computer Engineering Advanced Research Institute in Virginia Tech University, Va, USA. Her research interests focus on intelligent information processing, machine learning and its application to diseaserelated bioinformatics, image processing, radar automatic target recognition, and pattern recognition.

Li Gao was born in China in 1978. She received her B.S. degree and M.S. degree from the Beijing Institute of Technology, Beijing, China, in 2001 and 2004. She is studying for her Ph.D. degree in signal and information processing in the Institute of Acoustics, CAS, Beijing, China. Her current research interests include image/video processing, multimedia signal processing, and pattern recognization.
Feng Zhao was born in China in 1974. He received his M.S. degree from School of Computer Science and Engineering, Xidian University, Xi'an, China, in 2005. Currently, he is studying for his Ph.D. degree in signal and information processing from Xidian University. His research interests include intelligent signal and information processing.

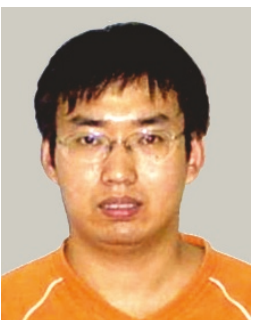

